\title{
On the Solution of Quadratic Nonlinear Integral Equation with Different Singular Kernels
}

\author{
M. Basseem ${ }^{1}$ and Ahmad Alalyani ${ }^{2}{ }^{2}$ \\ ${ }^{1}$ Sinai University, Arish, Egypt \\ ${ }^{2}$ Albaha University, Albaha, Saudi Arabia \\ Correspondence should be addressed to Ahmad Alalyani; aalalyani@hotmail.com
}

Received 30 July 2020; Accepted 14 October 2020; Published 19 November 2020

Academic Editor: Mariusz Michta

Copyright (c) 2020 M. Basseem and Ahmad Alalyani. This is an open access article distributed under the Creative Commons Attribution License, which permits unrestricted use, distribution, and reproduction in any medium, provided the original work is properly cited.

\begin{abstract}
All the previous authors discussed the quadratic equation only with continuous kernels by different methods. In this paper, we introduce a mixed nonlinear quadratic integral equation (MQNLIE) with singular kernel in a logarithmic form and Carleman type. An existence and uniqueness of MQNLIE are discussed. A quadrature method is applied to obtain a system of nonlinear integral equation (NLIE), and then the Toeplitz matrix method (TMM) and Nystrom method are used to have a nonlinear algebraic system (NLAS). The Newton-Raphson method is applied to solve the obtained NLAS. Some numerical examples are considered, and its estimated errors are computed, in each method, by using Maple 18 software.
\end{abstract}

\section{Introduction}

Integral equations of various types and kinds play an important role in several mathematical problems modelling. Analytical solutions of integral equations, however, neither exist nor simple to find, so several numerical methods have been developed for finding the solutions of integral equations. The quadratic equation provides an important tool for modelling many numerical phenomena, bio-mathematical problems, and process engineering. In genetics, it represents the reproduction equation, through which events affecting the cells can be predicted. Gripenberg [1] studied the existence and the uniqueness of a bounded continuous solution to the following integral equation of product type:

$$
x(t)=k\left[p(t)+\int_{0}^{t} A(t-s) x(s) \mathrm{d} s\right]\left[g(t)+\int_{0}^{t} B(t-s) x(s) \mathrm{d} s\right]
$$

which arises in the study of the spread of an infectious disease that does not induce permanent immunity.

Abdou and Basseem [2] used Chebyshev polynomial in solving mixed integral equation in position and time using spectral relationships. Javidi and Golbabai [3] solved NLFIE by the modified homotopy perturbation method. Alipanah and Esmaeili [4] used radial basis function to find a solution of two-dimensional FIE. Bernstein's method is used to solve
VIE by Maleknejad et al. [5]. The Toeplitz matrix method is used to solve NLIE of Hammerstein by Abdou et al. [6]. Orsi [7] used the product Nystrom method to get the solution of NVIE when its kernel takes a logarithmic form and Carleman function. The degenerate kernel method is discussed in three-dimensional NLIE by Basseem $[8,9]$. Guoqiang et al. [10] obtained numerically the solution of two-dimensional NVIE by collocation and iterated collocation 
methods. Brunner et al. [11] introduced a class of methods to obtain numerically the solution of Abel integral equation. Abdou and Raad [12] used the Adomian decomposition method for solving quadratic NLIE. The radial basis function method with collocation scheme for solving quadratic integral equation of Urysohn's type is described by Avazzadeh [13]. Assaria et al. used meshless methods for solving NLIE (see [14-17]).
In this paper, a new problem in a product type of mixed integral equation with singular kernel is considered. The existence and uniqueness of its solution are discussed.

The quadratic method is applied to obtain a NLS of FIE, and then the Toeplitz matrix method or Nystrom method is used to obtain a NLAS which is solved numerically by the Newton-Raphson method.

Consider the QNLE

$$
\gamma(x, t, \varphi(x, t))-\lambda \int_{0}^{t} V(t, \tau) \varphi(x, t) \mathrm{d} \tau \int_{-1}^{1} k(|x-y|) \varphi(y, t) \mathrm{d} y=f(x, t),
$$

where $V(|t, \tau|)$ is a continuous function of time, belongs to the class $C([0, T],[0, T]), t, \tau \in[0, T], T<1$. The singular kernel of position $k(|x-y|)$ takes many different forms. The given function $f(x, t)$ is in the space $C[0, T] \times L_{2}[-1,1]$. $\gamma(x, t, \varphi(x, t))$ is a given nonlinear function of the unknown function $\varphi(x, t)$. The constant $\lambda$ has many physical meanings.

\section{Existence and Uniqueness}

In order to guarantee the existence of a unique solution of equation (2), assume

(1) The discontinuous kernel of equation (2) verifies

$$
\left\{\int_{-1}^{1} \int_{-1}^{1} k^{2}(x-y) \mathrm{d} x \mathrm{~d} y\right\}^{(1 / 2)}=C .
$$

(2) The positive kernel of time is continuous and satisfies

$$
\max _{0 \leq t, \tau \leq T} V(|t-\tau|) \leq M, \quad \forall t, \tau \in[0, T] .
$$

(3) The given continuous function $f(x, t) \in L_{2}$ $[-1,1] \times C[0, T]$ and its norm is defined as

$$
\|f(x, t)\|=\max _{0 \leq t \leq T} \int_{0}^{t} f(x, \tau) \mathrm{d} \tau\left[\int_{-1}^{1} f^{2}(x, t) \mathrm{d} x\right]^{(1 / 2)} \leq H .
$$

(4) The function $\gamma(x, t, \varphi(x, t))$ satisfies

(i) $\|\gamma(x, t, \varphi(x, t))\|=\max _{0 \leq t \leq T} \int_{0}^{t} \gamma(x, \tau, \varphi(x, \tau)) \mathrm{d} \tau$
$\left[\int_{-1}^{1} \gamma^{2}(x, t, \varphi(x, t)) \mathrm{d} x\right]^{1 / 2} \leq E$ and $\|\varphi\|=D$, where $\mathrm{D}$ and $\mathrm{E}$ are constants.

(ii) For any two functions $\varphi_{1}$ and $\varphi_{2}, \gamma(x, t, \varphi(x, t))$ satisfies Lipchitz condition which is

$$
\left\|\gamma\left(x, t, \varphi_{1}(x, t)\right)-\gamma\left(x, t, \varphi_{2}(x, t)\right)\right\| \leq N\left\|\varphi_{1}(x, t)-\varphi_{2}(x, t)\right\| .
$$

\section{Integral Operator of MQNIE}

Eq. (2) can be written in the operator form as

$$
\Gamma=\Upsilon \varphi+F
$$

where

$$
\Upsilon \varphi=\lambda \int_{0}^{t} V(t, \tau) \varphi(x, t) \mathrm{d} \tau \int_{-1}^{1} k(|x-y|) \varphi(y, t) \mathrm{d} y .
$$

$\Gamma$ and $F$ are the Nemytskii operator generated by the functions $\gamma\left(x, t, \varphi_{2}(x, t)\right)$ and $f(x, t)$, respectively.

Theorem 1. The solution of equation (2) exists and is unique under the condition $|\lambda|<(1 / M C)$.

The proof of this theorem can be deduced after the following discussion.

Lemma 1. The operator $Y$ is bounded.

Proof. Since

$$
\|\Upsilon \varphi\| \leq|\lambda| \int_{0}^{t}|V(t, \tau) \varphi(x, \tau)| \mathrm{d} \tau \int_{-1}^{1}|k(|x-y|) \varphi(y, t)| \mathrm{d} y,
$$

applying Cauchy-Schwarz inequality, we have

$$
\|\Upsilon \varphi\| \leq|\lambda| M C D .
$$

Lemma 2. The operator $Y$ is continuous.

Proof. We assume two functions $\varphi_{n}$ and $\varphi_{m}$ satisfy equation (2), and then we get

$$
\begin{aligned}
\left\|\Upsilon \varphi_{n}-\Upsilon \varphi_{m}\right\| \leq & |\lambda| \int_{0}^{t}|V(t, \tau)|\left|\varphi_{n}(x, t)-\varphi_{m}(x, t)\right| \mathrm{d} \tau \\
& \cdot \int_{-1}^{1}|k(|x-y|)|\left|\varphi_{n}(x, t)-\varphi_{m}(x, t)\right| \mathrm{d} y .
\end{aligned}
$$


Applying Cauchy-Schwarz inequality, we have

$$
\left\|\Upsilon \varphi_{n}-\Upsilon \varphi_{m}\right\| \leq|\lambda| M C\left\|\varphi_{n}(x, t)-\varphi_{m}(x, t)\right\|,
$$

whenever $\left\|\varphi_{n}(x, t)-\varphi_{m}(x, t)\right\| \longrightarrow 0$, one can deduce $\left\|\Upsilon \varphi_{n}-\Upsilon \varphi_{m}\right\| \longrightarrow 0$, which proves the continuity of the operator.

Moreover, under the condition $|\lambda|<(1 / M C)$, the operator $\Upsilon$ is a contraction mapping, and by fixed point theorem, equation (2) has a unique solution in the space $L_{2}$ $[-1,1] \times C[0, T]$.

\section{Quadratic Numerical Method (See $[18]$ )}

To obtain a system of NLIE, divide the time interval $[0, T]$ as

$$
0=t_{0}<t_{1}<t_{2}<\ldots<t_{l}=T .
$$

Let $t=t_{i}$, then equation (2) becomes

$$
\gamma\left(x, t_{i}, \varphi\left(x, t_{i}\right)\right)-\lambda \int_{0}^{t_{i}} V\left(t_{i}, \tau\right) \varphi(x, t) \mathrm{d} \tau \int_{-1}^{1} k(|x-y|) \varphi\left(y, t_{i}\right) \mathrm{d} y=f\left(x, t_{i}\right) .
$$

Applying the quadrature rule, equation (14) reduces to

$$
\gamma\left(x, t_{i}, \varphi\left(x, t_{i}\right)\right)-\lambda \sum_{j=0}^{i} \omega_{j} V_{i, j} \varphi_{j}(x) \int_{-1}^{1} k(|x-y|) \varphi_{i}(y) \mathrm{d} y=f_{i}(x)
$$

where

$$
\omega_{j}= \begin{cases}\frac{h}{2}, & j=0, i, \\ h, & \text { otherwise. }\end{cases}
$$

$$
\begin{aligned}
\varphi_{i}(x) & =\varphi\left(x, t_{i}\right), \\
V_{i, j} & =V\left(t_{i}, t_{j}\right), \\
\gamma_{i}\left(x, \varphi_{i}(x)\right) & =\gamma\left(x, t_{i}, \varphi_{i}(x)\right),
\end{aligned}
$$

we get

Using the notation

$$
\gamma_{i}\left(x, \varphi_{i}(x)\right)-\lambda \int_{-1}^{1} k(|x-y|) \varphi_{i}(y) \mathrm{d} y\left[\frac{h}{2} V_{i, i} \varphi_{i}(x)+\sum_{j=0}^{i-1} \omega_{j} V_{i, j} \varphi_{j}(x)\right]=f_{i}(x),
$$

which is the system of NLIE can be solved by two different methods, namely, Toeplitz matrix method and Nystrom method.

\subsection{Algebraic System of NLIE. Consider}

$$
\Gamma \gamma\left(\varphi_{i}, x\right)=\lambda U \varphi_{i}(x) \cdot V \varphi_{i}(x)+f_{i}(x),
$$

where

$$
U \varphi_{i}(x)=\int_{-1}^{1} k|x-y| \varphi_{i}(y) \mathrm{d} y
$$

and

$$
V \varphi_{i}(x)=\sum_{j=0}^{i} \omega_{j} V_{i, j} \varphi_{j}(x)
$$

In order to guarantee the existence of a unique solution of an algebraic system of NIE, we assume the following conditions: (i) $\max f_{i}(x) \leq H^{*}$

(ii) $\sum_{j=0}^{i} \max \left|\omega_{j} V_{i, j}\right| \leq M^{*}$

(iii) $\max _{i}\left|\gamma^{j}\left(x, \varphi_{i}(x)\right)\right| \leq E^{*}$

Hence, formula (19) has a unique solution under condition $\lambda<\left(1 / C M^{*}\right)$.

Definition 1. The estimate local error $R^{(1)}$ is determined by the following relation:

$$
\begin{aligned}
R^{(1)}= & \mid \int_{-1}^{1} k(|x-y|) \varphi(y, t) \mathrm{d} y \int_{0}^{t} V(t, \tau) \varphi(x, \tau) \mathrm{d} \tau \\
& -\int_{-1}^{1} k(|x-y|) \varphi_{i}(y) \mathrm{d} y \sum_{j=0}^{i} \omega_{j} V_{i, j} \varphi_{j}(x) \mid .
\end{aligned}
$$

\section{Toeplitz Matrix Method (See [6])}

We apply the TMM to have a nonlinear algebraic equation. For this, consider $h=(1 / N)$; therefore, 


$$
\int_{-1}^{1} k(|x-y|) \varphi_{i}(y) \mathrm{d} y=\sum_{n=-N}^{N-1} \int_{n h}^{n h+h} k(|x-y|) \varphi_{i}(y) \mathrm{d} y
$$

and

$$
\int_{n h}^{n h+h} k(|x-y|) \varphi_{i}(y) \mathrm{d} y \approx A_{n}(x) \varphi(n h)+B_{n}(x) \varphi(n h+h)+R .
$$

The functions $A_{n}(x)$ and $B_{n}(x)$ are arbitrary functions to be determined, and $R$ is the error term. In order to obtain the values of two functions, assume $\varphi(y)=\{1, y\}$. This yields a set of two equations in terms of two unknown functions. After ignoring the error term, equation (18) becomes

$$
\begin{aligned}
& \gamma_{i}\left(x, \varphi_{i}(x)\right)-\frac{h|\lambda|}{2} \sum_{n=-N}^{N} \xi_{n}(x) \varphi_{i}(n h) V_{i, i} \varphi_{i}(x) \\
& =|\lambda| \sum_{n=-N}^{N} \xi_{n}(x) \varphi_{i}(n h) \sum_{j=0}^{i-1} \omega_{j} V_{i, j} \varphi_{j}(x)+f_{i}(x) .
\end{aligned}
$$

Let $x=m h$, then using the following notation:

$$
\begin{aligned}
\varphi_{i}(x) & =\varphi_{i}(m h)=\varphi_{i m}, \\
\xi_{n}(x) & =\xi_{n m}, \\
f_{i}(x) & =f_{i m} \\
\gamma_{i}\left(x, \varphi_{i}(x)\right) & =\gamma_{i m}\left(\varphi_{i m}\right),
\end{aligned}
$$

equation (25) becomes

$$
\begin{aligned}
\gamma_{i m}\left(\varphi_{i m}\right)-\frac{h|\lambda| V_{i i}}{2} \sum_{n=-N}^{N} \xi_{n m} \varphi_{i n} \varphi_{i m} \\
=|\lambda| \sum_{n=-N}^{N} \xi_{n m} \varphi_{i n} \sum_{j=0}^{i-1} \omega_{j} V_{i, j} \varphi_{j m}+f_{i m},
\end{aligned}
$$

where

$$
\xi_{n m}= \begin{cases}A_{-n}(m h), & n=-N, \\ A_{n}(m h)+B_{n-1}(m h), & -N<n<N, \\ B_{n-1}(m h), & n=N .\end{cases}
$$

Equation (27) represents that the NLAS can be solved using the Newton-Raphson method.

Definition 2. The Toeplitz matrix method is said to be convergent of order $r$ in the interval $[-1,1]$, if and only if, for sufficient large $N$, there exists a constant $D>0$ independent of $N$ such that

$$
\left\|\varphi(x)-\varphi_{N}(x)\right\| \leq D N^{-r} .
$$

Definition 3. The estimate local error $R^{(2)}$ is determined by the following relation:

$$
R^{(2)}=\left|\int_{-1}^{1} k(|x-y|) \varphi_{i}(y) \mathrm{d} y \sum_{j=0}^{i} \omega_{j} V_{i, j} \varphi_{j}(x)-\sum_{n=-N}^{N} \xi_{n m} \varphi_{i n} \sum_{j=0}^{i} \omega_{j} V_{i, j} \varphi_{j m}\right| .
$$

5.1. Existence and Uniqueness of NLAS. In order to guarantee the existence of a unique solution of a NLAS, we assume the following conditions:

$$
\begin{aligned}
& \text { (i) } \sup _{n, m}\left|\sum_{n=-N}^{N} \xi_{n m}\right| \leq C^{*} \\
& \text { (ii) } \sup _{i, m}\left|f_{i m}\right| \leq H^{* *}
\end{aligned}
$$

(iii) $\sup _{i, n}\left|\gamma_{i}(n h, \varphi(n h))\right| \leq E^{* *}$

Definition 4. The estimate local error $\mathrm{R}^{(\mathrm{T})}$ is determined by the following relation:

$$
R^{(T)}=\left|\int_{-1}^{1} k(|x-y|) \varphi(y, t) \mathrm{d} y \int_{0}^{t} V(t, \tau) \varphi(x, t) \mathrm{d} \tau-\sum_{n=-N}^{N} \xi_{n m} \varphi_{i n} \sum_{j=0}^{i} \omega_{j} V_{i, j} \varphi_{j m}\right|,
$$

where $R^{(T)} \leq R^{(1)}+R^{(2)}$.

\section{Nystrom Method (See [7])}

Here, by using the product integration, we approximate the integral part of equation (18) by a suitable Lagrange interpolation polynomial. For this, let $x=x_{m}$, and the integral part can be written as

$$
\int_{-1}^{1} k\left(x_{m}, y\right) \varphi_{i}(y) \mathrm{d} y=\sum_{n=0}^{N} \mathfrak{\Im}_{m, n} \varphi_{i}\left(y_{n}\right)=\sum_{n=0}^{((N-2) / 2)} \int_{y_{2 n}}^{y_{2 n+2}} k\left(x_{m}, y\right) \varphi_{i}(y) \mathrm{d} y,
$$


where $x_{m}=y_{m}=-1+m h, m=1,2,3, \ldots, N$ with $h=2 / N$ and $N$ is even number.

Approximate the nonsingular part of the integral $\varphi(y)$ over each interval $\left[y_{2 n}, y_{2 n+2}\right]$ by the Lagrange interpolation polynomial at the points $2 n, 2 n+1$, and $2 n+2$. Therefore, equation (32) becomes

$$
\begin{aligned}
\int_{-1}^{1} k\left(x_{m}, y\right) \varphi_{i}(y) \mathrm{d} y= & \sum_{n=0}^{N-2 / 2} \int_{y_{2 n}}^{y_{2 n+2}} k\left(x_{m}, y\right)\left[\frac{\left(y_{2 n+1}-y\right)\left(y_{2 n+2}-y\right)}{\left(y_{2 n+1}-y_{2 n}\right)\left(y_{2 n+2}-y_{2 n}\right)} \varphi_{i}\left(y_{2 n}\right)+\frac{\left(y_{2 n}-y\right)\left(y_{2 n+2}-y\right)}{\left(y_{2 n}-y_{2 n+1}\right)\left(y_{2 n}-y_{2 n+1}\right)} \varphi_{i}\left(y_{2 n+1}\right)\right. \\
& \left.+\frac{\left(y_{2 n}-y\right)\left(y_{2 n+1}-y\right)}{\left(y_{2 n}-y_{2 n+2}\right)\left(y_{2 n+1}-y_{2 n+2}\right)} \varphi_{i}\left(y_{2 n+2}\right)\right] \mathrm{d} y .
\end{aligned}
$$

Comparing equations (32) and (33), we deduce

$$
\begin{aligned}
\mathfrak{J}_{m, 0} & =\frac{1}{2 h^{2}} \int_{y_{0}}^{y_{2}} k\left(x_{m}, y\right)\left(y_{1}-y\right)\left(y_{2}-y\right) \mathrm{d} y, \\
\mathfrak{J}_{m, 2 n+1} & =\frac{1}{h^{2}} \int_{y_{2 n}}^{y_{2 n+2}} k\left(x_{m}, y\right)\left(y-y_{2 n}\right)\left(y_{2 n+2}-y\right) \mathrm{d} y, \\
\mathfrak{J}_{m, 2 n} & =\frac{1}{2 h^{2}}\left[\int_{y_{2 n}}^{y_{2 n+2}} k\left(x_{m}, y\right)\left(y_{2 n+1}-y\right)\left(y_{2 n+2}-y\right) \mathrm{d} y+\int_{y_{2 n-2}}^{y_{2 n}} k\left(x_{m}, y\right)\left(y-y_{2 n-1}\right)\left(y-y_{2 n-2}\right) \mathrm{d} y\right], \\
\mathfrak{J}_{m, N} & =\frac{1}{2 h^{2}} \int_{y_{N-2}}^{y_{N}} k\left(x_{m}, y\right)\left(y-y_{N-2}\right)\left(y-y_{N-1}\right) \mathrm{d} y .
\end{aligned}
$$

Introduce the following notations:

$$
\begin{aligned}
& \alpha_{n}\left(y_{m}\right)=\frac{1}{2 h^{2}} \int_{y_{2 n-2}}^{y_{2 n}} k\left(x_{m}, y\right)\left(y-y_{2 n-2}\right)\left(y-y_{2 n-1}\right) \mathrm{d} y, \\
& \beta_{n}\left(y_{m}\right)=\frac{1}{2 h^{2}} \int_{y_{2 n-2}}^{y_{2 n}} k\left(x_{m}, y\right)\left(y_{2 n-1}-y\right)\left(y_{2 n}-y\right) \mathrm{d} y,
\end{aligned}
$$

and

$$
\zeta_{n}\left(y_{m}\right)=\frac{1}{2 h^{2}} \int_{y_{2 n-2}}^{y_{2 n}} k\left(x_{m}, y\right)\left(y-y_{2 n-2}\right)\left(y_{2 n}-y\right) \mathrm{d} y .
$$

Then,

$$
\begin{aligned}
\mathfrak{J}_{m, 0} & =\beta_{1}\left(x_{m}\right), \\
\mathfrak{\Im}_{m, 2 n+1} & =2 \zeta_{n+1}\left(x_{m}\right), \\
\mathfrak{J}_{m, 2 n} & =\alpha_{n}\left(x_{m}\right)+\beta_{n+1}\left(x_{m}\right), \\
\mathfrak{J}_{m, N} & =\alpha_{(N / 2)}\left(x_{m}\right) .
\end{aligned}
$$

By substituting in equation (18), we get

$$
\gamma_{i} m\left(\varphi_{i m}\right)-\lambda \sum_{n=0}^{N} \mathfrak{\Im}_{m, n} \varphi_{i}\left(y_{n}\right)\left[\frac{h}{2} V_{i, i} \varphi_{i m}+\sum_{j=0}^{i-1} \omega_{j} V_{i, j} \varphi_{j m}\right]=f_{i m}
$$

where equation (38) represents the NAS in which its existence and uniqueness can be easily shown as in the previous section.

Definition 5. The Nystrom method is said to be convergent of order $r$ in the interval $[-1,1]$, if and only if, for sufficient large $N$, there exists a constant $K>0$ independent of $N$ such that

$$
\left\|\varphi(x)-\varphi_{N}(x)\right\| \leq K N^{-r}
$$

\section{Numerical Examples}

7.1. Example 1. Consider the equation

$$
\gamma(\varphi, x, t)-\lambda \int_{0}^{t} V(t, \tau) \varphi(x, \tau) \mathrm{d} \tau \cdot \int_{-1}^{1} k|x-y| \varphi(y, t) \mathrm{d} y \mathrm{~d} t=f(x, t),
$$


where $f(x, t)$ is given by putting $\varphi(x, t)=x^{2} t^{2}$ as an exact value with $\gamma=\varphi^{2}, V(t, \tau)=(t-\tau)^{2}$, and

$$
k(|x-y|)=\left\{\begin{array}{l}
\ln |y-x|, \\
|x-y|^{\nu}, \quad 0<\nu<1 .
\end{array}\right.
$$

(1) The following table is selected among a large amount of data to compare between the exact solution and its numerical solution in the case of logarithmic kernel for both of the previous methods in some points in the region $x \in[0,1]$ and for different values of time $T=\{0.009,0.02,0.8\}$.

(2) In the following table, we compare between the Toeplitz matrix method and Nystrom method for different $\nu$ and fixed time $T=0.3$ in Carleman kernel form.

7.2. Example 2. In the next example, the Nystrom method and Toeplitz matrix method are used with fixed time $T=$ 0.4 and the position interval is divided with $N=2,4,8,16,32$ points. The rate of errors is evaluated using the formula

$$
\text { Rate }=\log _{2} \frac{\operatorname{Error}(2 N)}{\operatorname{Error}(N)} .
$$

The negative sign means that by increasing $N$, the error decreases (see Table 3).

$$
\begin{aligned}
& e^{\varphi(x, t)}-\lambda \int_{0}^{t}(t-\tau)^{2} \varphi(x, \tau) \mathrm{d} \tau \cdot \int_{-1}^{1} \ln |x-y| \varphi(y, t) \mathrm{d} y \mathrm{~d} t \\
& =f(x, t),
\end{aligned}
$$

where $f(x, t)$ is given by setting $\phi(x, t)=x t$ as an exact value.

\section{General Conclusion}

From the above tables and our numerical results, we can deduce the following:

(1) The estimated error increases by time, where its mean errors by using Toeplitz and Nystrom methods, when $T=0.02$, are $1.876 \times 10^{-12}$ and $2.191 \times 10^{-12}$, respectively, while, its mean errors when $T=0.8$ are $8.565 \times 10^{-6}$ and $3.192 \times 10^{-5}$, respectively.

(2) The Toeplitz matrix method is comparatively better than the Nystrom method for different kernels (see Tables 1 and 2).

(3) By increasing $N$, the error is extremely stable in both methods, but in the Toeplitz matrix method, the error almost decreases by increasing in $N$, where the convergence rate with $+v e$ sign means the increasing of errors, while its -ve sign means the errors decreasing (see Table 3).

(4) In Carleman kernel form, the estimated error decreases in small values of $v$, where its mean errors take $1.816 \times 10^{-8}$ when $v=0.07$, while it takes
TABle 1: Comparison between the Toeplitz matrix method and Nystrom method in different time when kernel takes logarithmic form.

\begin{tabular}{cccccc}
\hline & \multicolumn{3}{c}{ Toeplitz matrix method } & \multicolumn{2}{c}{ Nystrom method } \\
$T$ & $x$ & $\varphi$ & Error & $\varphi$ & Error \\
\hline & -0.87 & 0.0000613 & $2.630 \times 10^{-15}$ & 0.0000613 & $4.950 \times 10^{-15}$ \\
& -0.37 & 0.0000111 & $2.157 \times 10^{-15}$ & 0.0000111 & $8.298 \times 10^{-15}$ \\
0.009 & 0.13 & 0.0000015 & $1.637 \times 10^{-7}$ & 0.0000015 & $1.642 \times 10^{-7}$ \\
& 0.63 & 0.0000321 & $1.219 \times 10^{-14}$ & 0.0000321 & $4.992 \times 10^{-15}$ \\
& 0.9 & 0.0000656 & $5.469 \times 10^{-15}$ & 0.0000656 & $1.139 \times 10^{-14}$ \\
\hline & -0.87 & 0.0003028 & $1.179 \times 10^{-13}$ & 0.0003028 & $4.435 \times 10^{-13}$ \\
& -0.37 & 0.0000548 & $5.055 \times 10^{-14}$ & 0.0000548 & $2.952 \times 10^{-13}$ \\
0.02 & 0.13 & 0.0000068 & $9.062 \times 10^{-12}$ & 0.0000068 & $9.582 \times 10^{-12}$ \\
& 0.63 & 0.0001588 & $1.417 \times 10^{-13}$ & 0.0001588 & $3.315 \times 10^{-13}$ \\
& 0.9 & 0.0003240 & $6.330 \times 10^{-15}$ & 0.0003240 & $3.040 \times 10^{-13}$ \\
\hline & -0.87 & 0.4844307 & $1.468 \times 10^{-5}$ & 0.4844625 & $4.647 \times 10^{-5}$ \\
& -0.37 & 0.0876232 & $7.192 \times 10^{-6}$ & 0.0876449 & $2.889 \times 10^{-5}$ \\
0.8 & 0.13 & 0.0108114 & $4.647 \times 10^{-6}$ & 0.0108321 & $1.606 \times 10^{-5}$ \\
& 0.63 & 0.2540051 & $1.094 \times 10^{-5}$ & 0.2540541 & $3.807 \times 10^{-5}$ \\
& 0.9 & 0.5183947 & $5.367 \times 10^{-6}$ & 0.5184301 & $3.009 \times 10^{-5}$ \\
\hline
\end{tabular}

TABLE 2: Errors in the Toeplitz matrix method and Nystrom method in different values of $v$ in Carleman kernel form.

\begin{tabular}{cccc}
\hline & & Toeplitz matrix method & Nystrom method \\
$\nu$ & $x$ & Error & Error \\
\hline \multirow{4}{*}{0.07} & -0.87 & $2.665 \times 10^{-8}$ & $3.198 \times 10^{-8}$ \\
& -0.37 & $2.218 \times 10^{-8}$ & $2.205 \times 10^{-8}$ \\
& 0.13 & $1.439 \times 10^{-8}$ & $1.734 \times 10^{-8}$ \\
& 0.63 & $1.204 \times 10^{-8}$ & $1.563 \times 10^{-8}$ \\
& 0.9 & $1.556 \times 10^{-8}$ & $2.184 \times 10^{-8}$ \\
\hline & -0.87 & $4.191 \times 10^{-8}$ & $5.629 \times 10^{-8}$ \\
0.17 & -0.37 & $2.924 \times 10^{-8}$ & $2.716 \times 10^{-8}$ \\
& 0.13 & $9.517 \times 10^{-9}$ & $1.726 \times 10^{-8}$ \\
& 0.63 & $9.449 \times 10^{-10}$ & $1.125 \times 10^{-8}$ \\
& 0.9 & $1.209 \times 10^{-8}$ & $3.042 \times 10^{-8}$ \\
\hline & -0.87 & $1.352 \times 10^{-7}$ & $2.110 \times 10^{-7}$ \\
0.47 & -0.37 & $6.733 \times 10^{-8}$ & $4.547 \times 10^{-8}$ \\
& 0.13 & $1.876 \times 10^{-8}$ & $1.748 \times 10^{-8}$ \\
& 0.63 & $6.492 \times 10^{-8}$ & $3.365 \times 10^{-10}$ \\
& 0.9 & $1.004 \times 10^{-8}$ & $1.268 \times 10^{-7}$ \\
\hline
\end{tabular}

TABLE 3: Convergence rate in both methods with fixed time $T=0.4$.

\begin{tabular}{lcccc}
\hline & \multicolumn{2}{c}{ Toeplitz matrix method } & \multicolumn{2}{c}{ Nystrom method } \\
$\mathrm{N}$ & Mean error & Rate & Mean error & Rate \\
\hline 2 & $6.641 \times 10^{-6}$ & - & $7.144 \times 10^{-6}$ & - \\
4 & $6.298 \times 10^{-6}$ & -0.0765067 & $1.079 \times 10^{-5}$ & 0.5956999 \\
8 & $6.482 \times 10^{-6}$ & 0.0415453 & $1.110 \times 10^{-5}$ & 0.0408648 \\
16 & $5.691 \times 10^{-6}$ & -0.1877568 & $1.102 \times 10^{-5}$ & -0.0104355 \\
32 & $5.556 \times 10^{-6}$ & -0.0346356 & $1.096 \times 10^{-5}$ & -0.0078764 \\
\hline
\end{tabular}

$5.925 \times 10^{-8}$ when $v=0.47$ by using the Toeplitz matrix method (see Table 2).

\section{Data Availability}

The authors confirm that the data supporting the findings of this study are included within the article. 


\section{Conflicts of Interest}

The authors declare that they have no conflicts of interest regarding the publication of this paper.

\section{Acknowledgments}

The authors expresses their sincere gratitude to Professor M. A. Abdou for his continuous and constructive interest during this work.

\section{References}

[1] G. Gripenberg, "On some epidemic models," Quarterly of Applied Mathematics, vol. 39, no. 3, pp. 317-327, 1981.

[2] M. A. Abdou and M. Basseem, "Solution of mixed integral equation in position and time using spectral relationships," Journal of the Association of Arab Universities for Basic and Applied Sciences, vol. 23, no. 1, pp. 52-56, 2017.

[3] M. Javidi and A. Golbabai, "Modified homotopy perturbation method for solving non-linear Fredholm integral equations," Chaos, Solitons \& Fractals, vol. 40, no. 3, pp. 1408-1412, 2009.

[4] A. Alipanah and S. Esmaeili, "Numerical solution of the twodimensional Fredholm integral equations using Gaussian radial basis function," Journal of Computational and Applied Mathematics, vol. 235, no. 18, pp. 5342-5347, 2011.

[5] K. Maleknejad, E. Hashemizadeh, and R. Ezzati, "A new approach to the numerical solution of Volterra integral equations by using Bernstein's approximation," Communications in Nonlinear Science and Numerical Simulation, vol. 16, no. 2, pp. 647-655, 2011.

[6] M. A. Abdou, M. M. El-Borai, and M. M. El-Kojok, "Toeplitz matrix method and nonlinear integral equation of Hammerstein type," Journal of Computational and Applied Mathematics, vol. 223, no. 2, pp. 765-776, 2009.

[7] A. P. Orsi, "Product integration for Volterra integral equations of the second kind with weakly singular kernels," Mathematics of Computation, vol. 56, no. 216, pp. 1201-1212, 1996.

[8] M. Basseem, "Degenerate kernel method for three dimension nonlinear integral equation of the second kind," Universal Journal of Integral Equations, vol. 3, pp. 61-66, 2015.

[9] M. Basseem, "Degenerate method in mixed nonlinear three dimensions integral equation," Alexandria Engineering Journal, vol. 58, no. 1, pp. 387-392, 2019.

[10] H. Guoqiang, K. Hayami, K. Sugihara, and W. Jiong, "Extrapolation method of iterated collocation solution for twodimensional nonlinear volterra integral equations," Applied Mathematics and Computation, vol. 112, no. 1, pp. 49-61, 2000.

[11] H. Brunner, M. R. Crisci, E. Russo, and A. Vecchio, "A family of methods for Abel integral equations of the second kind," Journal of Computational and Applied Mathematics, vol. 34, no. 2, pp. 211-219, 1991.

[12] M. A. Abdou and S. A. Raad, "New numerical approach for the nonlinear quadratic integral equations," Journal of Computational and Theoretical Nanoscience, vol. 13, no. 10, pp. 6435-6439, 2016.

[13] Z. Avazzadeh, "A numerical approach for solving quadratic integral equations of Urysohn's type using radial basis function," Journal of Applied \& Computational Mathematics, vol. 1 , no. 4 , p. 116,2012

[14] P. Assaria and M. Dehghan, "A meshless local discrete Galerkin (MLDG) scheme for numerically solving two- dimensional nonlinear Volterra integral equations," Applied Mathematics and Computation, vol. 350, pp. 249-265, 2019.

[15] P. Assaria, "The numerical solution of Fredholm-Hammerstein integral equations by combining the collocation method and radial basis functions," Filomat, vol. 33, no. 3, 2019.

[16] P. Assaria and M. Dehghan, "Solving a class of nonlinear boundary integral equations based on the meshless local discrete Galerkin (MLDG) method," Applied Numerical Mathematics, vol. 123, pp. 137-158, 2018.

[17] P. Assaria, “Thin plate spline Galerkin scheme for numerically solving nonlinear weakly singular Fredholm integral equations," Journal-Applicable Analysis, vol. 123, no. 11, pp. 2064-2084, 2019.

[18] L. M. Delves and J. L. Mohamed, Computational Methods for Integral Equations, Cambridge University Press, Cambridge, UK, 1985. 